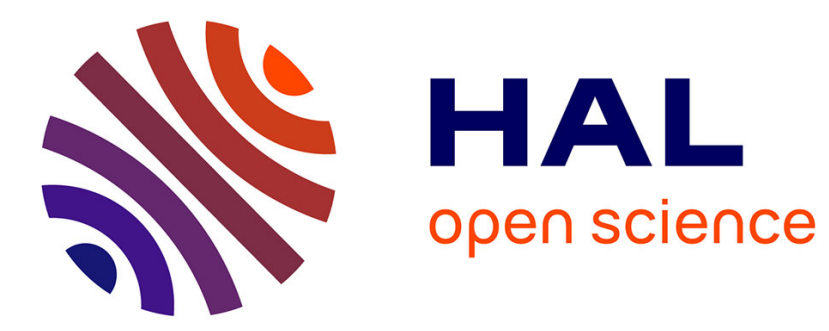

\title{
Error estimate for the Finite Volume Scheme
}

Benoit Merlet, Julien Vovelle

\section{To cite this version:}

Benoît Merlet, Julien Vovelle. Error estimate for the Finite Volume Scheme. Numerische Mathematik, 2007, 106 (1), pp.129-155. 10.1007/s00211-006-0053-y . hal-00008660v3

\section{HAL Id: hal-00008660 \\ https://hal.science/hal-00008660v3}

Submitted on 22 Mar 2019

HAL is a multi-disciplinary open access archive for the deposit and dissemination of scientific research documents, whether they are published or not. The documents may come from teaching and research institutions in France or abroad, or from public or private research centers.
L'archive ouverte pluridisciplinaire HAL, est destinée au dépôt et à la diffusion de documents scientifiques de niveau recherche, publiés ou non, émanant des établissements d'enseignement et de recherche français ou étrangers, des laboratoires publics ou privés. 


\title{
Error estimate for Finite Volume scheme
}

\author{
Benoît Merlet and Julien Vovelle
}

Summary. We study the convergence of a Finite Volume scheme for the linear advection equation with a Lipschitz divergence-free speed in $\mathbf{R}^{d}$. We prove a $h^{1 / 2}$-error estimate in the $L^{\infty}\left(0, t ; L^{1}\right)$-norm for $B V$ data. This result was expected from numerical experiments and is optimal.

Keywords : scalar conservation laws, advection equation, Finite Volume method, error estimate

Mathematics Subject Classification : 35L65, 65M15

\section{Introduction}

The Finite Volume method is well adapted to the computation of the solution of pdes which are conservation (or balance) laws, for the reason that it respects the property of conservation of the pde under study. The mathematical analysis of the application of the Finite Volume method to hyperbolic first-order conservation laws can be dated from the mid sixties (see [TS62] for example). Concerning the specific problem of the estimate of the rate of convergence of the method, the first result is due to Kuztnetsov [Kuz76], who proves that this rate of convergence in $L^{\infty}\left(0, t ; L^{1}\right)$ is of order $h^{1 / 2}$, where $h$ is the size of the mesh, provided that the initial data is in $B V$ and that the mesh is a structured cartesian grid. Ever since, several studies and results have come to supplement the error estimate of Kuznetsov. Before describing them, let us emphasize two points:

1. The analysis of the speed of convergence of the Finite Volume method is distinct from the analysis of the order of the method. In the analysis of the speed of convergence of the method, general data (e.g. $B V$ data) are considered. Indeed, here, the problem is to show that the Finite Volume method behaves well regarding the approximation of the continuous evolution problem in all his features (in particular the creation and the transport of discontinuities). On the other hand, in the analysis of the order of the method, restrictions on the regularity of the data are of no importance: see the recent work of Bouche, Ghidaglia, Pascal [BGP05] on that purpose.

2. If, numerically, the speed of convergence of the Finite Volume method applied to firstorder conservation laws is observed to be (at least) of order $h^{1 / 2}$ in the $L^{\infty}\left(0, t ; L^{1}\right)$-norm, whether the mesh is structured or is not, the preexisting theoretical and rigorous proofs of this result appears to be strongly related to the structure of the mesh. 
Indeed, in the case where the mesh is unstructured (what we call a structured mesh is a cartesian mesh with identical cells but this can be slightly relaxed [CGY98]), the result of Kuznetsov has been extended, but to the price of a fall in the order of the error estimate. Indeed, $h^{1 / 4}$ error estimate in the $L^{\infty}\left(0, t ; L^{1}\right)$-norm for the Finite Volume method applied to hyperbolic conservation laws on unstructured meshes has been proved by Cockburn, Coquel, Lefloch [CCL94], Vila [Vil94] and Eymard, Gallouët, Herbin [EGH00] for the Cauchy Problem ( $h^{1 / 6}$ error estimate for the Cauchy-Dirichlet Problem [OV04]). We emphasize the fact that numerical tests give an order $h^{1 / 2}$ for structured as well as unstructured meshes; still, concerning these latter, numerical analysis did not manage to give the rigorous proof of the order $h^{1 / 2}$ : there is an upper limit at the order $h^{1 / 4}$.

In this paper, we consider the case where the conservation law is linear. More precisely, we consider the linear advection problem with a Lipschitz divergence-free speed.

In the case of $H^{1}$-data, optimal (with respect to the order of the error estimate) results already exist. Under a strict CFL condition, Després [Des04c] shows an $h^{1 / 2}$-error estimate in the $L_{t}^{\infty} L_{x}^{2}$-norm for the upwind Finite Volume method applied to the linear advection equation with constant speed. (The author deals with the particular case of 2D triangular meshes but extensions to higher dimension and general polyhedral meshes are harmless). His technique is based on the study of the dissipation of the consistency error by the scheme and the adaptation of the Lax Theorem (see also [Des04a]). Roughly speaking, the consistency error created at time $n \delta t$ is of order 1 but of order $1 / \sqrt{q+1}$ after $q$ time steps of the scheme.

On the other hand, we refer to the work of Vila and Villedieu [VV03], who prove, again under a strict CFL condition, an $h^{1 / 2}$-error estimate in the $L_{l o c}^{2}$ space-time norm for the approximation of Friedrichs hyperbolic systems with $H^{1}$ data by energy estimates (they consider explicit in time Finite Volume schemes, for implicit schemes in the case of scalar advection equation, see, as they underline it, the result of Johnson and Pitkäranta [JP86] who show an $h^{1 / 2}$-error estimate in the $L^{2}$ space-time norm for $H^{1}$ data).

For an initial data $u_{0} \in B V\left(\mathbf{R}^{d}\right)$, we prove in Theorem 2 the expected $h^{1 / 2}$-error estimate in the $L_{t}^{\infty} L_{x}^{1}$ norm, under a strict CFL condition in the general case and, under a sharp CFL condition if the speed is independent of $t$. This result is optimal [TT95, Şab97] and in the context of unstructured meshes and $B V$-data, this is the first optimal result.

In our proof of Theorem 2, we first show that it is sufficient to consider initial data which are characteristic functions. Then we use the same ingredients as Vila and Villedieu in [VV03]. The approximate solution satisfies the weak formulation of the problem up to an error term corresponding to the consistency error of the scheme. Roughly speaking, this error is bounded via the Cauchy-Schwarz inequality by a $H^{1}\left(\mathbf{R}^{d} \times[0, t]\right)$-like seminorm of the discrete solution denoted $E_{h}\left(u_{0}, t\right)$. A large error means that the scheme is very dissipative and consequently, that the approximate solution is smooth, so $E_{h}\left(u_{0}, t\right)$ is small. The $h^{1 / 2}$-estimate follows from the equilibrium between these contradictory constraints.

The paper is divided into six parts: first we continue this introduction by describing the linear advection problem, the Finite Volume scheme, our results and we give the main 
lines of the proof. In Section 2 we recall some classical results on the Finite Volume scheme. In Section 3 we introduce the weak formulation satisfied by the approximate solution. Then we reduce the study to initial data which are characteristic functions and we build the test function used in the weak formulation. In Section 4, we prove some Energy estimates. The first and second part of Theorem 2 are proved in Sections 5, 6 respectively.

\section{Notations}

If $(X, \mu)$ is a measurable set with finite (positive) measure and $\phi \in L^{1}(X)$, we denote the mean of $\phi$ over $X$ by

$$
f_{X} \phi d \mu:=\frac{1}{\mu(X)} \int_{X} \phi d \mu
$$

If $X$ is a set, $\mathbf{1}_{X}(x)=1$ if $x \in X, 0$ if $x \notin X$.

The set of functions with bounded variation in $\mathbf{R}^{d}$ is the set of $L^{1}$ functions with bounded Radon measures as derivatives:

$$
B V\left(\mathbf{R}^{d}\right):=\left\{u \in L^{1}\left(\mathbf{R}^{d}\right) ; \sup _{\varphi \in \mathcal{C}_{c}^{\infty}\left(\mathbf{R}^{d}, \mathbf{R}^{d}\right),\|\varphi\|_{\infty} \leq 1}\left|\int_{U} u(x) \operatorname{div} \varphi(x) d x\right|<+\infty\right\}
$$

where $\|\varphi\|_{\infty}:=\left\|\sqrt{\varphi_{1}^{2}+\cdots+\varphi_{d}^{2}}\right\|_{L^{\infty}\left(\mathbf{R}^{d}\right)}$ for $\varphi \in \mathcal{C}_{c}^{\infty}\left(\mathbf{R}^{d}, \mathbf{R}^{d}\right)$ and div is the divergence operator. The total variation of $u \in B V\left(\mathbf{R}^{d}\right)$ is given by

$$
\|u\|_{T V}:=\sup _{\varphi \in \mathcal{C}_{c}^{\infty}\left(\mathbf{R}^{d}, \mathbf{R}^{d}\right),\|\varphi\|_{\infty} \leq 1}\left|\int_{\mathbf{R}^{d}} u(x) \operatorname{div} \varphi(x) d x\right| .
$$

\subsection{The linear advection equation}

We consider the linear advection problem in $\mathbf{R}^{d}$ :

$$
\begin{cases}u_{t}+\operatorname{div}(V u)=0, & x \in \mathbf{R}^{d}, t \in \mathbf{R}_{+}, \\ u(x, 0)=u_{0}(x), \quad x \in \Omega,\end{cases}
$$

where we suppose that $V \in W^{1, \infty}\left(\mathbf{R}^{d} \times \mathbf{R}_{+}, \mathbf{R}^{d}\right)$ satisfies

$$
\operatorname{div} V(\cdot, t)=0 \quad \forall t \in \mathbf{R}_{+} .
$$

The problem (1.1) has a solution for $u_{0} \in L_{l o c}^{1}\left(\mathbf{R}^{d}\right)$; for the purpose of the error estimates, we will consider initial data in $B V\left(\mathbf{R}^{d}\right)$. 
Theorem 1. For every $u_{0} \in L_{\text {loc }}^{1}\left(\mathbf{R}^{d}\right)$, the problem (1.1) admits a unique weak solution $u$, in the sense that $u \in L_{\text {loc }}^{1}\left(\mathbf{R}_{+} \times \mathbf{R}^{d}\right)$ and: for every $\phi \in \mathcal{C}_{c}^{\infty}\left(\mathbf{R}^{d} \times \mathbf{R}_{+}\right)$,

$$
\int_{\mathbf{R}_{+}} \int_{\mathbf{R}^{d}} u\left(\phi_{t}+V \cdot \nabla \phi\right) d x d t+\int_{\mathbf{R}^{d}} u_{0} \phi(x, 0) d x=0 .
$$

Moreover, we have

$$
u(x, t)=u_{0}(X(x, t)), \quad \forall(x, t) \in \mathbf{R}^{d} \times \mathbf{R}_{+},
$$

where $X \in C^{1}\left(\mathbf{R}^{d} \times \mathbf{R}_{+}, \mathbf{R}^{d}\right)$ is such that, for every $t \geq 0, X(\cdot, t): \mathbf{R}^{d} \rightarrow \mathbf{R}^{d}$ is one to one and onto.

Let $Y(\cdot, t):=X(\cdot, t)^{-1}$, then for every $T \geq 0, X-I d_{\mathbf{R}_{x}^{d}}$ and $Y-I d_{\mathbf{R}_{x}^{d}}$ belong to $W^{1, \infty}\left(\mathbf{R}^{d} \times\right.$ $[0, T])$. More precisely, there exists $C_{0} \geq 1$ only depending on $T,\|V\|_{W^{1, \infty}}$ and $d$, such that, $\forall x \in \mathbf{R}^{d}, \forall t \in[0, T]$,

$$
\begin{aligned}
& \|X(x, t)-x\| \leq C_{0} t, \quad\|\nabla X(x, t)\| \leq C_{0}, \quad\left\|\partial_{t} X(x, t)\right\| \leq C_{0}, \\
& \|Y(x, t)-x\| \leq C_{0} t, \quad\|\nabla Y(x, t)\| \leq C_{0}, \quad\left\|\partial_{t} Y(x, t)\right\| \leq C_{0} .
\end{aligned}
$$

Finally, for every $t \geq 0, X(\cdot, t)$ preserves the Lebesgue measure $\lambda$ on $\mathbf{R}^{d}$, i.e:

$$
\lambda(X(E, t))=\lambda(E), \quad \text { for every Borel subset } E \text { of } \mathbf{R}^{d} .
$$

Proof of Theorem 1 All the results cited in the Theorem follow from the characteristic formula: $u(x, t)=u_{0}(X(x, t))$ where $X(\cdot, t)=Y(\cdot, t)^{-1}$ and $Y$ solves the Cauchy Problem $\partial_{t} Y(x, t)=V(Y(x, t), t), Y(x, 0)=x$. To prove the estimates on $X$, we notice that $X(x, t)=Z(0 ; x, t)$ where $Z(\tau ; x, t)$ denotes the solution of the Cauchy Problem

$$
\left\{\begin{array}{l}
\frac{d Z}{d \tau}(\tau ; x, t)=V(Z(\tau ; x, t), \tau), \quad \tau \in[0, t], \\
Z(t ; x, t)=x
\end{array}\right.
$$

We only give the sketch of the proof: global existences for $Y$ and $Z$ and the desired estimates follow from the Cauchy-Lipschitz Theorem and the Gronwall Lemma. Besides, by (1.2), the flow preserves the Lebesgue measure on $\mathbf{R}^{d}$. Existence for (1.1) follows from the characteristic formula and, by an argument of duality, uniqueness also.

If $u_{0} \in L_{l o c}^{1}\left(\mathbf{R}^{d}\right)$, the solution $u$ belongs to $C\left(\mathbf{R}_{+}, L_{l o c}^{1}\left(\mathbf{R}^{d}\right)\right)$. If the derivatives of $u_{0}$ are bounded Radon measures, we have a more precise result. Namely, using the estimates above, we obtain

Corollary 1.1. Let $T \geq 0$, there exists a constant $C_{0} \geq 1$ depending on $\|V\|_{W^{1, \infty}}, T$ and $d$ such that, if $u_{0} \in B V\left(\mathbf{R}^{d}\right)$ :

$$
\begin{aligned}
\|u(\cdot, s)-u(\cdot, t)\|_{L^{1}} & \leq C_{0}\left\|u_{0}\right\|_{T V}|s-t|, & 0 \leq s, t \leq T \\
\|u(\cdot, t)\|_{T V} & \leq C_{0}\left\|u_{0}\right\|_{T V}, & 0 \leq t \leq T
\end{aligned}
$$


The following result is a direct consequence of the conservative property (1.7). The case $f(v)=v^{2}$ will be crucial in the proof of the main result of this paper.

Corollary 1.2. Let $f: \mathbf{R} \rightarrow \mathbf{R}$ be a measurable function and $u_{0} \in L_{\text {loc }}^{1}\left(\mathbf{R}^{d}\right)$ such that $f \circ u_{0} \in L^{1}\left(\mathbf{R}^{d}\right)$. Then the quantity $\int_{\mathbf{R}^{d}} f(u(\cdot, t))$ is constant.

\subsection{Finite Volume scheme}

The Finite Volume scheme which approximates (1.1) is defined on a mesh $\mathcal{T}$ which is a family of closed connected polygonal subsets with disjoint interiors covering $\mathbf{R}^{d}$; the time half-line is meshed by regular cells of size $\delta t>0$. We also suppose that the partition $\mathcal{T}$ satisfies the following properties: the common interface of two control volumes is included in an hyperplane of $\mathbf{R}^{d}$ and

$$
\text { there exists } \alpha>0 \text { such that }\left\{\begin{array}{l}
\alpha h^{d} \leq|K| \\
|\partial K| \leq \alpha^{-1} h^{d-1}, \forall K \in \mathcal{T}
\end{array}\right.
$$

where $h$ is the size of the mesh: $h:=\sup \{\operatorname{diam}(K), K \in \mathcal{T}\},|K|$ is the $d$-dimensional Lebesgue measure of $K$ and $|\partial K|$ is the $(d-1)$-dimensional Lebesgue measure of $\partial K$. If $K$ and $L$ are two control volumes having a common edge, we say that $L$ is a neighbor of $K$ and denote (quite abusively) $L \in \partial K$. We also denote $K$ । $L$ the common edge and $\mathbf{n}_{K L}$ the unit normal to $K ı L$ pointing outward $K$. We set

$$
V_{K L}^{n}:=\int_{K \backslash L} \int_{n \delta t}^{(n+1) \delta t} V \cdot \mathbf{n}_{K L}
$$

We denote by $K_{n}:=K \times[n \delta t,(n+1) \delta t)$ a generic space-time cell, by $\mathcal{M}:=\mathcal{T} \times \mathbb{N}$ the space-time mesh and by $\partial K_{n}^{-}$the set

$$
\partial K_{n}^{-}:=\left\{L \in \partial K, V_{K L}^{n}<0\right\} .
$$

In the same way we set $K_{\iota} L_{n}:=K_{।} L \times[n \delta t,(n+1) \delta t)$.

We will assume that the so called Courant-Friedrich-Levy condition is satisfied:

$$
\sum_{L \in \partial K_{n}^{-}} \delta t\left|V_{K L}^{n}\right| \leq(1-\xi)|K|, \quad \forall K_{n} \in \mathcal{M}, \quad \text { for some } \xi \in[0,1) .
$$

Remark 1.1. Under condition (1.8), the CFL condition (1.9) holds as soon as

$$
\|V\|_{\infty} \delta t \leq(1-\xi) \alpha^{-2} h
$$

Notice that if $\|V\|_{\infty}$ is small, we may choose a large time step $\delta t$. In order to avoid the occurrence of terms with factor $\delta t h^{-1}$ in our estimates, we add to (1.9) the following condition: there exists $c_{0} \geq 0$ such that

$$
\delta t \leq c_{0} h
$$


The Finite Volume scheme with explicit time-discretization is defined by the following set of equations:

$$
\begin{array}{r}
u_{K}^{0}=f_{K} u_{0}(x) d x, \quad \forall K \in \mathcal{T}, \\
\frac{u_{K}^{n+1}-u_{K}^{n}}{\delta t}+\frac{1}{|K|} \sum_{L \in \partial K_{n}^{-}} V_{K L}^{n}\left(u_{L}^{n}-u_{K}^{n}\right)=0, \quad \forall K_{n} \in \mathcal{M} .
\end{array}
$$

We then denote by $u_{h}$ the approximate solution of (1.1) defined by the Finite Volume scheme:

$$
u_{h}(x, t)=u_{K}^{n}, \quad \forall(x, t) \in K_{n}
$$

\section{Main results}

From now on, we assume that $V \in W^{1, \infty}\left(\mathbf{R}^{d} \times \mathbf{R}_{+}, \mathbf{R}^{d}\right)$ satisfies (1.2). We fix a mesh $\mathcal{T}$ of mesh-size $h>0$ satisfying the uniformity conditions (1.8). We also fix a time step $\delta t$ such that the CFL conditions (1.9)-(1.10) hold.

We fix two times $0 \leq t \leq T$ and we assume that $t=(N+1) \delta t$ for some $N \in \mathbb{N}$ (so that $\left.u_{h}(\cdot, t)=\sum_{K \in \mathcal{T}} u_{K}^{N+1} \mathbf{1}_{K}\right)$. In order to avoid the occurrences of $h t^{-1}$ terms in the estimates we assume that

$$
h \leq c_{1} t, \quad \text { for some } c_{1} \geq 0 .
$$

In the sequel, given an initial condition $u_{0} \in B V\left(\mathbf{R}^{d}\right)$, the function $u \in C\left(\mathbf{R}_{+}, L^{1}\left(\mathbf{R}^{d}\right)\right)$ denotes the exact solution to (1.1) and $u_{h}$ its numerical approximation obtained by the upwind Finite Volume method (1.11)-(1.12)-(1.13).

In the results and in the proofs, $C_{0} \geq 1$ is the constant introduced in Theorem 1 and Corollary 1.1; this constant only depends on $T,\|V\|_{W^{1, \infty}}$ and $d$. The letter $C$ denotes various constants which are non decreasing functions of $\alpha^{-1}, c_{0}, c_{1},\|V\|_{W^{1, \infty}}$ and $d$ but do not depend on $h, \delta t, \xi, u_{0}, t$ or $T$.

Finally, before stating the main results of the paper, we set

$$
\mathcal{M}_{N}:=\left\{K_{n} \in \mathcal{M}: 0 \leq n \leq N\right\}
$$

and we introduce the quantities

$$
\begin{aligned}
E_{h}\left(u_{0}, t\right) & :=\sum_{K_{n} \in \mathcal{M}_{N}}|K|\left|u_{K}^{n+1}-u_{K}^{n}\right|^{2}+\sum_{K_{n} \in \mathcal{M}_{N}} \sum_{L \in \partial K_{n}^{-}} \delta t\left|V_{K L}^{n}\right|\left|u_{L}^{n}-u_{K}^{n}\right|^{2}, \\
\mathcal{E}_{h}\left(u_{0}, t\right) & :=\left\|u_{h}(\cdot, 0)\right\|_{L^{2}}^{2}-\left\|u_{h}(\cdot, t)\right\|_{L^{2}}^{2} .
\end{aligned}
$$

Remark 1.2. Notice that $h^{-2} E_{h}\left(u_{0}, t\right)$ is a discrete version of the quantity

$$
\int_{\mathbf{R}^{d} \times[0, t]}\left(\left|\partial_{t} u_{h}(x, s)\right|^{2}+\sum_{i=1}^{d}\left|V_{i}(x, s)\right|\left|\partial_{x_{i}} u_{h}(x, s)\right|^{2}\right) d x d s .
$$


We have the following error estimate:

Theorem 2. Let $u_{0}$ in $B V\left(\mathbf{R}^{d}\right)$. Under a strict $C F L$ condition ((1.9) with $\left.\xi>0\right)$, we have:

$$
\left\|u(\cdot, t)-u_{h}(\cdot, t)\right\|_{L^{1}} \leq C C_{0}^{d+2} \xi_{*}^{-1}\left\|u_{0}\right\|_{T V}\left(t^{1 / 2} h^{1 / 2}+\xi_{*}^{1 / 2} t h\right),
$$

with $\xi_{*}=\xi$.

Moreover, if $V$ does not depend on the time variable, the estimate is valid with $\xi_{*}=1$ uniformly in $\xi \in[0,1)$.

This result deserves some comments.

Remark 1.3. If the speed does not depend on $x: V(x, s):=\mathbf{V}(s)$, we have $X(x, s)=$ $x-\int_{0}^{s} \mathbf{V}(r) d r$ and we can choose $C_{0}=d^{1 / 2}+\|\mathbf{V}\|_{\infty}$ (which does not depend on $T$ ) in Theorem 1 and Corollary 1.1.

Remark 1.4. In the proof of the first part of Theorem 2, the dependency on $\xi$ only appears in Lemma 5.1.

Remark 1.5 (On the $B V$-norm of the approximate solution). The possible irregularity of the mesh is an obstacle to the existence of uniform (with respect to $h$ ) bound on the $L_{t}^{1} B V_{x}$-norm

$$
\sum_{K_{n} \in \mathcal{M}_{N}} \sum_{L \in \partial K_{n}^{-}} \delta t|K| L|| u_{L}^{n}-u_{K}^{n} \mid
$$

of the approximate solution $u_{h}$. A counter-example to such a result has been given by B. Després in [Des04b]. However, a uniform bound on the weaker norm (notice the weights $\left.V_{K L}^{n}\right)$

$$
Q_{h}\left(u_{0}, t\right):=\sum_{K_{n} \in \mathcal{M}_{N}} \sum_{L \in \partial K_{n}^{-}} \delta t\left|V_{K L}^{n}\right|\left|u_{L}^{n}-u_{K}^{n}\right|
$$

would be enough to prove the error estimate (1.15) by use of the techniques of Kuznetsov [Kuz76] (and, therefore, regardless whether the conservation law under consideration is linear, the remark here is true). This uniform bound on $Q_{h}\left(u_{0}, t\right)$ is observed in numerical experiments and is probably true. However, in this paper, we do not prove this claim and it remains an open problem, even in the linear setting.

Interpolating between the $h^{1 / 2}$-error estimate in the $L_{t}^{\infty} L_{x}^{2}$-norm of [Des04c] and the estimate of Theorem 2, we obtain, under a strict CFL condition, a $h^{1 / 2}$-error estimate in the $L_{t}^{\infty} L_{x}^{p}$-norm for data in $W^{1, p}\left(\mathbf{R}^{d}\right), 1 \leq p \leq 2$.

The analogue result for initial data in $W^{1, p}\left(\mathbf{R}^{d}\right), 2 \leq p \leq \infty$, requires different techniques and will be addressed in a subsequent paper.

\subsection{Sketch of the proof of Theorem 2}

We only sketch the proof of the first part of the Theorem; besides we do not exhibit the dependency on $t$ and $\xi$ of the error estimates (the constants $C$ may depend on $t, T$ or $\xi$ ). 
To prove Theorem 2, we use the fact that the difference between the exact and the approximate solutions satisfies the weak formulation (Lemma 3.1):

$$
\int_{\mathbf{R}^{d} \times \mathbf{R}_{+}}\left(u-u_{h}\right)\left(\phi_{t}+V \cdot \nabla \phi\right)+\int_{\mathbf{R}}\left(u-u_{h}\right)(\cdot, 0) \phi(x, 0) d x=\left(\mu_{h}+\nu_{h}\right)(\phi),
$$

for every $\phi \in C_{c}^{1}\left(\mathbf{R}^{d} \times \mathbf{R}_{+}\right)$, where the error term $\left(\mu_{h}+\nu_{h}\right)(\phi)$ encompasses the consistency error of the Finite Volume approximation and depends on the approximate solution.

We then notice that, by linearity of the equations, and subsequent principle of superposition, we may suppose that the initial data $u_{0} \in B V\left(\mathbf{R}^{d}\right)$ is the characteristic function $u_{0}=\mathbf{1}_{A}$ of a set $A$ with finite perimeter. The norm $\left\|u_{0}\right\|_{B V}$ is equal to the perimeter of $A$. In fact, we show that we can also reduce the study to the case where $A$ does not contain very thin parts so that, in particular, the volume of the $h^{1 / 2}$-neighborhood of $\partial A$

$$
A_{0}:=\left\{x \in \mathbf{R}^{d}: d(x, \partial A) \leq h^{1 / 2}\right\} .
$$

is bounded by $C|\partial A| h^{1 / 2}$. At this stage, we build a function $\phi_{0} \in C^{1}\left(\mathbf{R}^{d},[-1,1]\right)$ such that $\phi_{0} \equiv 1$ on $A \backslash A_{0}, \phi_{0} \equiv-1$ on $A^{c} \backslash A_{0}$ and $\left\|\nabla \phi_{0}\right\|_{\infty} \leq C h^{-1 / 2}$. Then we set $\phi(x, s)=\phi_{0}(X(x, s)) \mathbf{1}_{[0, t]}(s)$ so that $\phi_{t}+V \cdot \phi=0$ on $\mathbf{R}^{d} \times[0, t]$. After a regularization process, the weak formulation yields

$$
\left\|\left(u_{h}-u\right)(\cdot, t)\right\|_{L^{1}} \leq C|\partial A| h^{1 / 2}+\left|\mu_{h}(\phi)\right|+\left|\nu_{h}(\phi)\right| .
$$

The term $\mu_{h}(\phi)$ is

$$
\mu_{h}(\phi)=\sum_{K \in \mathcal{M}_{N}}|K|\left|u_{K}^{n+1}-u_{K}^{n}\right|\left(f_{K_{n}} \phi-f_{K} \phi(\cdot,(n+1) \delta t)\right) .
$$

The purpose of the reduction to the case $u_{0}=\mathbf{1}_{A}$ is an accurate estimation of the term $\mu(\phi)$. Indeed, by definition of $\phi$, we have: for $0 \leq s \leq t, \phi(x, s)=1$ if $x \in X\left(A \backslash A_{0}, s\right)$ and $\phi(x, s)=-1$ if $x \in X\left(A^{c} \backslash A_{0}, s\right)$. More precisely, there exists $C>0$ such that, if

$$
d\left(K_{n}, \cup_{0 \leq s \leq t}(X(\partial A, s) \times\{s\}) \geq C\left(h^{1 / 2}+h+\delta t\right),\right.
$$

then $\phi$ is constant on the cell $K_{n}$. We denote by $\mathcal{M}_{N}^{1}$ the set of cells which do not satisfy this property; we have

$$
\mu_{h}(\phi)=\sum_{K \in \mathcal{M}_{N}^{1}}|K|\left|u_{K}^{n+1}-u_{K}^{n}\right|\left(f_{K_{n}} \phi-f_{K} \phi(\cdot,(n+1) \delta t)\right) .
$$

and the $(d+1)$-dimensional volume of the cells of $\mathcal{M}_{N}^{1}$ is bounded by $C|\partial A| h^{1 / 2}$ (Lemma 3.5). We use the Cauchy-Schwarz inequality and the estimate $\left\|\partial_{t} \phi\right\|_{\infty} \leq C h^{-1 / 2}$ to get

$$
\left|\mu_{h}(\phi)\right| \leq C E\left(u_{0}, t\right)^{1 / 2}|\partial A|^{1 / 2} h^{1 / 4} .
$$

A similar technique yields the same bound on $\nu_{h}(\phi)$. 
Thanks to the Energy estimate (Lemma 5.1) $E_{h}\left(u_{0}, t\right) \leq C \mathcal{E}_{h}\left(u_{0}, t\right)$, we get

$$
\left\|\left(u_{h}-u\right)(\cdot, t)\right\|_{L^{1}} \leq C\left(|\partial A| h^{1 / 2} \mathcal{E}_{h}\left(u_{0}, t\right)^{1 / 2}+|\partial A|^{1 / 2} h^{1 / 4}\right) .
$$

Finally, since the $L^{2}$-norm of the exact solution is conserved, we get (Lemma 4.1):

$$
\mathcal{E}_{h}\left(u_{0}, t\right) \leq 2\left\|\left(u_{h}-u\right)(\cdot, t)\right\|_{L^{1}} .
$$

Together with the preceding inequality, this yields successively $\mathcal{E}_{h}\left(u_{0}, t\right) \leq C|\partial A| h^{1 / 2}$ and $\left\|\left(u_{h}-u\right)(\cdot, t)\right\|_{L^{1}} \leq C|\partial A| h^{1 / 2}$.

\section{Classical results}

By continuity in $B V$ of the $L^{2}$-projection on the space of functions which are constant with respect to the mesh $\mathcal{T}$, we have:

Lemma 2.1. There exists a constant $c \geq 0$ only depending on $d$ such that for every $u_{0} \in B V\left(\mathbf{R}^{d}\right)$,

$$
\begin{aligned}
\left\|u_{h}(\cdot, 0)\right\|_{T V}=\frac{1}{2} \sum_{K \in \mathcal{M}, L \in \partial K}\left|K_{\mid} L\right|\left|u_{L}^{0}-u_{K}^{0}\right| & \leq c \alpha^{-3}\left\|u_{0}\right\|_{T V}, \\
\left\|u_{h}(\cdot, 0)-u_{0}\right\|_{L^{1}} & \leq c \alpha^{-1}\left\|u_{0}\right\|_{T V} h .
\end{aligned}
$$

Proof of Lemma 2.1: Using the weak density of $C_{c}^{1}\left(\mathbf{R}^{d}\right)$ in $B V\left(\mathbf{R}^{d}\right)$, we may suppose that $u_{0} \in C_{c}^{1}\left(\mathbf{R}^{d}\right)$. Let $K \in \mathcal{T}, L \in \partial K$. Since $|x-y| \leq 2 h$ for every $(x, y) \in K \times L$, we have

$$
\begin{aligned}
\left|u_{K}^{0}-u_{L}^{0}\right| & \leq \frac{1}{|K||L|} \int_{K} \int_{L}\left|u_{0}(x)-u_{0}(y)\right| d x d y \\
& \leq \frac{2 h}{|K||L|} \int_{K} \int_{L} \int_{0}^{1}\left|\nabla u_{0}((1-r) x+r y)\right| d r d x d y .
\end{aligned}
$$

Now we perform the change of variables $(x, y, r) \mapsto(w=x-y, z=(1-t) x+t y, r=r)$ (of Jacobian determinant equal to 1 ). We have

$$
\left|u_{K}^{0}-u_{L}^{0}\right| \leq \frac{2 h}{|K||L|} \int_{B\left(x_{K}, 2 h\right)}\left|\nabla u_{0}(z)\right|\left(\int_{0}^{1} \int_{\mathbf{R}^{d}} g(w, z, r) d w d r\right) d z
$$

where $x_{K} \in \mathbf{R}^{d}$ is the centroid of $K$, and $g$ is defined by $g(w, z, r)=1$ if $z+r w \in K$ and $z-(1-r) w \in L$, and $g(w, z, r)=0$ otherwise. For $(z, r) \in B\left(x_{K}, 2 h\right) \times[0,1]$, we have $\int_{\mathbf{R}^{d}} g(w, z, r) d w \leq 2^{d}|K|$ if $r \geq 1 / 2$ and $\int_{\mathbf{R}^{d}} g(w, z, r) d w \leq 2^{d}|L|$ if $r<1 / 2$. Finally, we obtain

$$
\left|u_{K}^{0}-u_{L}^{0}\right| \leq \frac{2^{d+1} \max (|K|,|L|) h}{|K||L|} \int_{B\left(x_{K}, 2 h\right)}\left|\nabla u_{0}(z)\right| d z
$$


We deduce from the inequalities (1.8) that for every $K \in \mathcal{T}$,

$$
\sum_{L \in \partial K}\left|K_{\mid} L\right|\left|u_{K}^{0}-u_{L}^{0}\right| \leq 2^{d+1} \alpha^{-2} \int_{B\left(x_{K}, 2 h\right)}\left|\nabla u_{0}(z)\right| d z .
$$

Summing on $K \in \mathcal{T}$, we get

$$
\sum_{K \in \mathcal{T}} \sum_{L \in \partial K}\left|K_{\mid} L\right|\left|u_{K}^{0}-u_{L}^{0}\right| \leq 2^{d+1} \alpha^{-2} \int_{\mathbf{R}^{d}}\left|\nabla u_{0}(z)\right| M_{2 h}(z) d z,
$$

where $M_{2 h}(z)$ is the cardinal of the set $\{K: d(K, z) \leq 2 h\}$. By (1.8), we have

$$
M_{2 h}(z) \alpha h^{d} \leq \sum_{K: d(K, z) \leq 2 h}|K| \leq|B(z, 3 h)| .
$$

Thus $M_{2 h}(z) \leq c \alpha^{-1}$ and we get the first part of the Lemma.

Let $K \in \mathcal{T}$. Similarly, we have

$$
\begin{aligned}
\int_{K}\left|u_{h}(\cdot, 0)-u_{0}\right| & \leq \frac{1}{|K|} \int_{K \times K}\left|u_{0}(x)-u_{0}(y)\right| d x d y \\
& \leq 2^{d} h \int_{B\left(x_{K}, h\right)}\left|\nabla u_{0}(z)\right| d z
\end{aligned}
$$

Summing on $K \in \mathcal{T}$ and using the fact that the cardinal of the set $\{K: d(K, z) \leq h\}$ is bounded by $c \alpha^{-1}$, we get the second estimate.

The free divergence assumption (1.2) leads to the following identity

Lemma 2.2. Under the hypothesis on the divergence of $V$ (1.2), one has

$$
\sum_{L \in \partial K_{n}^{-}} V_{K L}^{n}=\sum_{L: K \in \partial L_{n}^{-}} V_{L K}^{n} \quad \forall K_{n} \in \mathcal{M}
$$

\section{Monotony}

Under a CFL condition the Finite Volume scheme is order-preserving:

Proposition 2.1. Under condition (1.9), the linear application $\mathcal{L}:\left(u_{K}^{n}\right) \mapsto\left(u_{K}^{n+1}\right)$ defined by (1.12) is order-preserving and stable for the $L^{\infty}$-norm.

Proof of Proposition 2.1: Eq. (1.12) gives

$$
u_{K}^{n+1}=\left(1+\sum_{L \in \partial K_{n}^{-}} \frac{V_{K L}^{n} \delta t}{|K|}\right) u_{K}^{n}-\sum_{L \in \partial K_{n}^{-}} \frac{V_{K L}^{n} \delta t}{|K|} u_{L}^{n}
$$

i.e., under (1.9), $u_{K}^{n+1}$ is a convex combination of $u_{K}^{n},\left(u_{L}^{n}\right)_{L \in \partial K_{n}^{-}}$. The stability result follows from $\mathcal{L}(1)=1$. 


\section{$L^{1}$-stability}

From Lemma 2.2 it is not difficult to see that the quantity $\sum_{K \in \mathcal{T}}|K| u_{K}^{n}$ is conserved. By the Crandall-Tartar Lemma [CT80], this fact and Proposition 2.1 imply

Proposition 2.2. Under condition (1.9), the scheme $\mathcal{L}:\left(u_{K}^{n}\right) \mapsto\left(u_{K}^{n+1}\right)$ is stable for the $L^{1}$-norm:

$$
\sum_{K \in \mathcal{T}}|K|\left|u_{K}^{n+1}\right| \leq \sum_{K \in \mathcal{T}}|K|\left|u_{K}^{n}\right|, \quad \forall n \geq 0
$$

\section{Weak Formulation}

In the remainder of the paper, we assume that $u_{0} \in B V\left(\mathbf{R}^{d}\right)$, that the function $u \in$ $C\left(\mathbf{R}_{+}, L^{1}\left(\mathbf{R}^{d}\right)\right)$ is the exact solution to (1.1) and $u_{h}$ is the numerical approximation given by the scheme (1.11)-(1.12)-(1.13). We also assume that (1.8), the CFL conditions (1.9)(1.10) and the condition (1.14) are satisfied.

We intend to prove that $u_{h}$ satisfies (1.3) up to an error term.

Lemma 3.1. For every $\phi \in \mathcal{C}_{c}^{\infty}\left(\mathbf{R}^{d} \times[0,+\infty)\right)$,

$$
\begin{aligned}
\int_{\mathbf{R}_{+}} \int_{\mathbf{R}^{d}} u_{h}\left(\phi_{t}+V \cdot \nabla \phi\right)+\int_{\mathbf{R}^{d}} u_{h}(\cdot, 0) \phi(\cdot, 0)=\mu_{h}(\phi)+\nu_{h}(\phi), \\
\mu_{h}(\phi):=\sum_{K_{n} \in \mathcal{M}}|K|\left(u_{K}^{n+1}-u_{K}^{n}\right)\left(\langle\phi\rangle_{K}^{n}-\langle\phi\rangle_{K}((n+1) \delta t)\right), \\
\nu_{h}(\phi):=\sum_{K_{n} \in \mathcal{M}} \sum_{L \in \partial K_{n}^{-}} \delta t\left(u_{L}^{n}-u_{K}^{n}\right)\left(V_{K L}^{n}\langle\phi\rangle_{K}^{n}-|K \backslash L|\langle V \cdot \mathbf{n} \phi\rangle_{K L}^{n}\right),
\end{aligned}
$$

where

$$
\langle\phi\rangle_{K}^{n}:=f_{K_{n}} \phi, \quad\langle\phi\rangle_{K}(t):=f_{K} \phi(\cdot, t), \quad\langle V \cdot \mathbf{n} \phi\rangle_{K L}^{n}:=f_{K i L_{n}} \phi V \cdot \mathbf{n}_{K L} .
$$

Proof of Lemma 3.1: We develop the first term of the inequality and perform a discrete integration by parts:

$$
\begin{aligned}
\int_{0}^{\infty} & \int_{\mathbf{R}^{d}} u_{h}\left(\phi_{t}+V \cdot \nabla \phi\right) d x d t=\sum_{K_{n} \in \mathcal{M}}|K| \delta t u_{K}^{n} f_{K_{n}}\left(\phi_{t}+V \cdot \nabla \phi\right) d x d t \\
= & \sum_{K_{n} \in \mathcal{M}} u_{K}^{n}\left(|K|\left(\langle\phi\rangle_{K}((n+1) \delta t)-\langle\phi\rangle_{K}(n \delta t)\right)+\sum_{L \in \partial K} \delta t|K \backslash L|\langle V \cdot \mathbf{n} \phi\rangle_{K L}^{n}\right) \\
= & \sum_{K_{n} \in \mathcal{M}}\left(|K|\left(u_{K}^{n}-u_{K}^{n+1}\right)\langle\phi\rangle_{K}((n+1) \delta t)+\sum_{L \in \partial K} \delta t|K \backslash L|\langle V \cdot \mathbf{n} \phi\rangle_{K L}^{n} u_{K}^{n}\right) \\
& -\sum_{K_{n} \in \mathcal{M}}|K| \delta t u_{K}^{0}\langle\phi\rangle_{K}(0) .
\end{aligned}
$$


The last sum is equal to $\int_{\mathbf{R}^{d}} u_{h}(\cdot, 0) \phi(\cdot, 0)$. Rearranging the terms in the second sum and using the identity $\langle V \cdot \mathbf{n} \phi\rangle_{K L}^{n}+\langle V \cdot \mathbf{n} \phi\rangle_{L K}^{n}=0$, we compute: for every $n \geq 0$

$$
\sum_{K \in \mathcal{T}} \sum_{L \in \partial K}\left|K_{ı} L\right|\langle V \cdot \mathbf{n} \phi\rangle_{K L}^{n} u_{K}^{n}=\sum_{K \in \mathcal{T}} \sum_{L \in \partial K_{n}^{-}}\left|K_{ı} L\right|\langle V \cdot \mathbf{n} \phi\rangle_{K L}^{n}\left(u_{K}^{n}-u_{L}^{n}\right) .
$$

Plugging this identity and the definition of the scheme (1.12) in (3.4), we get (3.1).

We now assume that $u_{0} \in B V\left(\mathbf{R}^{d}\right)$. Before building the test function $\phi$, we show that we may reduce the study to more simple initial data.

Let $\mathcal{T}^{\prime}$ be the cartesian mesh

$$
\left\{t^{1 / 2} h^{1 / 2} \prod_{k=1}^{d}\left[p_{k}, p_{k}+1\right]:\left(p_{1}, \cdots, p_{d}\right) \in \mathbf{Z}^{d}\right\}
$$

The diameter of the cells is $h^{\prime}=d^{1 / 2} t^{1 / 2} h^{1 / 2}$; this mesh is uniform: conditions (1.8) are satisfied for $\alpha^{\prime}=\min \left(d^{-d / 2}, d^{(d-3) / 2} / 2\right)$. In particular, by Lemma 2.1, we have

$$
\left\|v_{0}\right\|_{T V} \leq C\left\|u_{0}\right\|_{T V}, \quad \quad\left\|v_{0}-u_{0}\right\|_{L^{1}} \leq C\left\|u_{0}\right\|_{T V} t^{1 / 2} h^{1 / 2}
$$

where $v_{0}$ is the projection defined by $v_{0}:=\sum_{K^{\prime} \in \mathcal{T}^{\prime}}\left(f_{K^{\prime}} u_{0}\right) \mathbf{1}_{K^{\prime}}$. These estimates, the $L^{1}$ stability of the scheme and of the evolution equation (1.1) imply that it is sufficient to prove Theorem 2 for the initial data $v_{0}$.

Lemma 3.2. [Fed69, Bre84] Let $v_{0} \in B V\left(\mathbf{R}^{d}\right)$. For every $\eta \in \mathbf{R}$, define

$$
\chi_{v_{0}}(x, \eta):=\left\{\begin{array}{r}
1 \quad \text { if } \quad 0<\eta<v_{0}(x) \\
-1 \quad \text { if } \quad v_{0}(x)<\eta<0 \\
0 \quad \text { in the other cases. }
\end{array}\right.
$$

We have $v_{0}=\int_{\mathbf{R}} \chi_{v_{0}}(\cdot, \eta) d \eta$, almost everywhere. Moreover,

$$
\begin{aligned}
\left\|v_{0}\right\|_{L^{1}} & =\int_{\mathbf{R}}\left\|\chi_{v_{0}}(\cdot, \eta)\right\|_{L^{1}} d \eta \\
\left\|v_{0}\right\|_{T V} & =\int_{\mathbf{R}}\left\|\chi_{v_{0}}(\cdot, \eta)\right\|_{T V} d \eta
\end{aligned}
$$

where the second identity is a consequence of the co-area formula for BV functions.

Applying this decomposition, we deduce from the linearity of the scheme and of the initial problem, that it is sufficient to prove the Theorem for any initial data $u_{0}$ which is a finite sum of characteristic functions $\mathbf{1}_{K^{\prime}}$.

Therefore, from now on, we suppose that $u_{0}=\mathbf{1}_{A}$ with $A=\cup_{1 \leq j \leq l} K_{j}^{\prime}$ and $K_{1}^{\prime}, \cdots, K_{l}^{\prime}$ are distinct elements of $\mathcal{T}^{\prime}$. 
Let us point out that

$$
\left\|u_{0}\right\|_{L^{1}}=|A|=l t^{d / 2} h^{d / 2},
$$

and since, the boundary $\partial A$ is a finite union of distinct edges denoted $K_{1}^{\prime} L_{1}^{\prime}, \cdots, K_{m}^{\prime} L_{m}^{\prime}$, we deduce from the definition of the Total Variation:

$$
\left\|u_{0}\right\|_{T V}=|\partial A|=\sum_{i=1}^{m}\left|K_{i}^{\prime}\right| L_{i}^{\prime} \mid=m t^{(d-1) / 2} h^{(d-1) / 2} .
$$

With the notations of Theorem 1 , we have $u=\mathbf{1}_{B}$ where $B \subset \mathbf{R}^{d} \times \mathbf{R}_{+}$is defined by

$$
B:=\bigcup_{s \geq 0} Y(A, s) \times\{s\} .
$$

We are now going to build a test function $\phi$ for (1.3) and (3.1). Let $\Gamma \in C^{\infty}(\mathbf{R}, \mathbf{R})$ such that $0 \leq \Gamma \leq 1, \Gamma(R)=0$ for $R \leq 1 / 3$ and $\Gamma(R)=1$ for $R \geq 2 / 3$. We define $\Phi_{0} \in \operatorname{Lip}\left(\mathbf{R}^{d}, \mathbf{R}\right)$ by

$$
\Phi_{0}(x):=\left\{\begin{array}{rll}
\Gamma\left(t^{-1 / 2} h^{-1 / 2} d(x, \partial A)\right) & \text { if } & x \in A, \\
-\Gamma\left(t^{-1 / 2} h^{-1 / 2} d(x, \partial A)\right) & \text { if } & x \in A^{c} .
\end{array}\right.
$$

Since $d(\cdot, \partial A)$ is a 1-Lipschitz continuous function, we have $\left\|\nabla \Phi_{0}\right\|_{\infty} \leq t^{-1 / 2} h^{-1 / 2}\left\|\Gamma^{\prime}\right\|_{\infty}$. Let us introduce a mollifier $\rho \in C_{c}^{\infty}\left(\mathbf{R}^{d}, \mathbf{R}_{+}\right)$such that $\int_{\mathbf{R}^{d}} \rho=1$ and $\operatorname{supp} \rho \subset B(0,1 / 3)$, we set $\phi_{0}:=t^{-d / 2} h^{-d / 2} \rho\left(t^{-1 / 2} h^{-1 / 2} \cdot\right) \star \Phi_{0}$. The function $\phi_{0}$ belongs to $C^{1}\left(\mathbf{R}^{d}\right)$ with the bound:

$$
\left\|\nabla \phi_{0}\right\|_{\infty} \leq t^{-1 / 2} h^{-1 / 2}\left\|\Gamma^{\prime}\right\|_{\infty}
$$

Moreover, we have

$$
x \in A \Longrightarrow \phi_{0}(x) \geq 0, \quad \text { and } \quad x \in A^{c} \Longrightarrow \phi_{0}(x) \leq 0,
$$

and if we split $\mathbf{R}^{d}$ in three disjoint subsets: $\mathbf{R}^{d}=\phi_{0}^{-1}(\{1\}) \cup \phi_{0}^{-1}((-1,1)) \cup \phi_{0}^{-1}(\{-1\})=$ : $A_{+} \cup A_{0} \cup A_{-}$, we have $u_{0} \equiv 1$ on $A_{+}, u_{0} \equiv 0$ on $A_{-}$and

$$
A_{0} \subset\left\{x: d(x, \partial A)<t^{1 / 2} h^{1 / 2}\right\} \subset \cup_{i=1}^{m} B\left(x_{i}, 1 / 2 t^{1 / 2} h^{1 / 2}\right),
$$

where $x_{i} \in \mathbf{R}^{d}$ is the centroid of $K_{i}^{\prime} \mid L_{i}^{\prime}, 1 \leq i \leq m$. Consequently the Lebesgue measure of $A_{0}$ satisfies:

$$
\left|A_{0}\right| \leq C m t^{d / 2} h^{d / 2}=C\left\|u_{0}\right\|_{T V} t^{1 / 2} h^{1 / 2}
$$

Finally, we define our test-function by $\phi(x, s):=\phi_{0}(X(x, s)) \mathbf{1}_{[0, t]}(s)$ for $(x, s) \in \mathbf{R}^{d} \times \mathbf{R}_{+}$, where $X$ is the mapping introduced in Theorem 1 . Since $\operatorname{div} V(\cdot, s) \equiv 0$ for every $s \geq 0$, we have

$$
\phi_{t}+V \cdot \nabla \phi=\phi_{t}+\operatorname{div}(V \phi)=0 \quad \text { in } \mathbf{R}^{d} \times(0, t),
$$


and from the estimate on $\nabla \phi_{0}$ above and on $\nabla X$ and $\partial_{t} X$ (1.5) in Theorem $1, \phi$ belongs to $C^{1}\left(\mathbf{R}^{d} \times[0, t]\right)$ and satisfies

$$
\begin{aligned}
\|\nabla \phi\|_{L^{\infty}\left(\mathbf{R}^{d} \times[0, T]\right)} & \leq C C_{0} t^{-1 / 2} h^{-1 / 2} \\
\left\|\partial_{t} \phi\right\|_{L^{\infty}\left(\mathbf{R}^{d} \times[0, T]\right)} & \leq C C_{0} t^{-1 / 2} h^{-1 / 2} .
\end{aligned}
$$

Moreover, since $u(x, s)=u_{0}(X(x, s))$, $\phi$ inherits the properties of $\phi_{0}$ : for $(x, s) \in \mathbf{R}^{d} \times \mathbf{R}_{+}$,

$$
u(x, s)=1 \Longrightarrow \phi(x, s) \geq 0, \quad \text { and } \quad u(x, s)=0 \Longrightarrow \phi(x, s) \leq 0 .
$$

Lemma 3.3. We have

$$
\int_{\mathbf{R}^{d}}\left(u-u_{h}\right)(\cdot, t) \phi(\cdot, t)-\int_{\mathbf{R}^{d}}\left(u-u_{h}\right)(\cdot, 0) \phi(\cdot, t)=\mu_{h}(\phi)+\nu_{h}(\phi) .
$$

Proof of Lemma 3.3: The test function $\phi$ is not compactly supported and not time differentiable at the time $t$. So it can not be used directly in (1.3), (3.1). First, remark that, since $u_{0}$ is compactly supported, there exists $R>0$ such that $\operatorname{supp} u_{h}(\cdot, s), \operatorname{supp} u(\cdot, s) \subset$ $B(0, R)$ for every $0 \leq s \leq t+\delta t$, thus $\phi$ may be replaced in (1.3), (3.1) by the compactly supported function $\phi \cdot \chi$ where $\chi \in C_{c}^{\infty}\left(\mathbf{R}^{d}\right)$ is such that $\chi \equiv 1$ on $B(0, R)$.

To overcome the non differentiability at $t$ we introduce a mollifying sequence to approximate the function $\mathbf{1}_{[0, t]}$. Let $\left(\psi_{q}\right)_{q \geq 1}$ be a sequence of $C_{c}^{\infty}\left(\mathbf{R}_{+}\right)$functions satisfying $\psi_{q} \equiv 1$ on $[0, t], \psi_{q} \equiv 0$ on $[t+\delta t / q,+\infty)$ and $0 \geq \psi_{q}^{\prime} \geq-2 q / \delta t$. We set $\phi_{q}(x, s):=\phi_{0}(X(x, s)) \psi_{q}(s)$ for every $(x, s) \in \mathbf{R}^{d} \times \mathbf{R}_{+}$and $q \geq 1$. The weak formulation (3.1) reads

$$
\int_{t}^{t+\delta t}\left(\int_{\mathbf{R}^{d}} u_{h}(x, s) \phi_{0}(X(x, s)) d x\right) \psi_{q}^{\prime}(s) d s+\int_{\mathbf{R}^{d}} u_{h}(\cdot, 0) \phi_{0}(\cdot, 0)=\mu_{h}\left(\phi_{q}\right)+\nu_{h}\left(\phi_{q}\right),
$$

The sequence of Radon measures $\left(\psi_{q}^{\prime}(s) d s\right)_{q}$ converges to $-\delta_{t}$, so the first term of the left hand side converges towards

$$
-\int_{\mathbf{R}^{d}} u_{h}(\cdot, t) \phi(\cdot, t)
$$

as $q$ tends to $+\infty$.

Clearly, for every $K_{n} \in \mathcal{M}$ and $L \in \partial K_{n}^{-}$, we have $\left(\left\langle\phi_{q}\right\rangle_{K}\right)_{q}^{n} \rightarrow\langle\phi\rangle_{K}^{n},\left(\left\langle V \cdot \mathbf{n} \phi_{q}\right\rangle_{K L}^{n}\right)_{q} \rightarrow$ $\langle V \cdot \mathbf{n} \phi\rangle_{K L}^{n}$ and $\left(\left\langle\phi_{q}\right\rangle_{K}(n \delta t)\right)_{q} \rightarrow\langle\phi\rangle_{K}(n \delta t)$. Passing to the limit on $q$, we get

$$
-\int_{\mathbf{R}^{d}} u_{h}(\cdot, t) \phi(\cdot, t)+\int_{\mathbf{R}^{d}} u_{h}(\cdot, 0) \phi_{0}=\mu_{h}(\phi)+\nu_{h}(\phi) .
$$

Similarly, we obtain from (1.3)

$$
-\int_{\mathbf{R}^{d}} u(\cdot, t) \phi(\cdot, t)+\int_{\mathbf{R}^{d}} u(\cdot, 0) \phi_{0}=0 .
$$

Subtracting these equalities, we get the result.

We relate the first term of the equality of Lemma 3.3 to the $L_{t}^{\infty} L_{x}^{1}$-norm of the error: 
Lemma 3.4. We have

$$
\left\|\left(u_{h}-u\right)(\cdot, t)\right\|_{L^{1}} \leq \int_{\mathbf{R}^{d}}\left(u-u_{h}\right)(\cdot, t) \phi(\cdot, t)+C\left\|u_{0}\right\|_{T V} t^{1 / 2} h^{1 / 2} .
$$

Proof of Lemma 3.4: Recall that from the monotony of the scheme, we have

$$
0 \leq u_{h}(x, s) \leq 1, \quad \forall(x, s) \in \mathbf{R}^{d} \times \mathbf{R}_{+} .
$$

Let us consider the disjoint decomposition $\mathbf{R}^{d}=X\left(A_{+}, t\right) \cup X\left(A_{0}, t\right) \cup X\left(A_{-}, t\right)$ and notice that (3.8), (3.11) imply that $\left(u(x, t)-u_{h}(x, t)\right) \phi(x, t) \geq 0$ for every $x \in \mathbf{R}^{d}$. In particular

$$
0 \leq \int_{X\left(A_{0}, t\right)}\left(u-u_{h}\right)(\cdot, t) \phi(\cdot, t) .
$$

Since $0 \leq u, u_{h} \leq 1$, we deduce from (3.5) and the conservation property (1.7) that

$$
\int_{X\left(A_{0}, t\right)}\left|\left(u_{h}-u\right)(\cdot, t)\right| \leq\left|X\left(A_{0}, t\right)\right|=\left|A_{0}\right| \leq C\left\|u_{0}\right\|_{T V} t^{1 / 2} h^{1 / 2} .
$$

Finally, using the identities $\phi(\cdot, t) \equiv 1, u(\cdot, t) \equiv 1$ on $X\left(A_{+}, t\right)$ and $\phi(\cdot, t) \equiv-1, u(\cdot, t) \equiv 0$ on $X\left(A_{-}, t\right)$ and the bounds $0 \leq u_{h} \leq 1$, we have

$$
\begin{aligned}
\int_{X\left(A_{+} \cup A_{-}, t\right)}\left|u_{h}(\cdot, t)-u(\cdot, t)\right| & =\int_{X\left(A_{+}, t\right)}\left(u-u_{h}\right)(\cdot, t)+\int_{X\left(A_{-}, t\right)}\left(u_{h}-u\right)(\cdot, t) \\
& =\int_{X\left(A_{+} \cup A_{-}, t\right)}\left(u-u_{h}\right)(\cdot, t) \phi(\cdot, t) .
\end{aligned}
$$

Summing up this equality and the two preceding estimates, we get (3.10).

Lemmas 3.3 and 3.4 and the second estimate of Lemma 2.1 (and $h \leq c_{1} t$ ) yield

$$
\left\|\left(u_{h}-u\right)(\cdot, t)\right\|_{L^{1}} \leq \mu_{h}(\phi)+\nu_{h}(\phi)+C\left\|u_{0}\right\|_{T V} t^{1 / 2} h^{1 / 2} .
$$

The first key idea to estimate $\mu_{h}(\phi)$ and $\nu_{h}(\phi)$ is to remark that most of the terms in the sums of (3.2), (3.3) vanish.

Lemma 3.5. There exists $\mathcal{M}_{N}^{1} \subset \mathcal{M}_{N}$ such that

$$
\begin{aligned}
\mu_{h}(\phi) & =\sum_{K_{n} \in \mathcal{M}_{N}^{1}}|K|\left(u_{K}^{n+1}-u_{K}^{n}\right)\left(\langle\phi\rangle_{K}^{n}-\langle\phi\rangle_{K}((n+1) \delta t)\right), \\
\nu_{h}(\phi) & =\sum_{K_{n} \in \mathcal{M}_{N}^{1}} \sum_{L \in \partial K_{n}^{-}} \delta t\left(u_{L}^{n}-u_{K}^{n}\right)\left(V_{K L}^{n}\langle\phi\rangle_{K}^{n}-|K । L|\langle V \cdot \mathbf{n} \phi\rangle_{K L}^{n}\right) .
\end{aligned}
$$

with the estimate

$$
\sum_{K_{n} \in \mathcal{M}_{N}^{1}} \delta t|K| \leq C C_{0}^{d}\left\|u_{0}\right\|_{T V} t^{3 / 2} h^{1 / 2}
$$


Proof of Lemma 3.5: Let us denote by $\mathcal{B}$ the open subset of $\mathbf{R}^{d} \times[0, t]$ of all the points $(x, s)$ such that $|\phi(x, s)|<1$. Let $K_{n} \in \mathcal{M}_{N}$ which does not intersect $\mathcal{B}$. In this case, $\phi$ is constant $(\phi \equiv 1$ or $\phi \equiv-1)$ on $K_{n}$ and we have

$$
\langle\phi\rangle_{K}^{n}=\langle\phi\rangle_{K}((n+1) \delta t) \quad \text { and } \quad V_{K L}^{n}\langle\phi\rangle_{K L}^{n}=|K| L \mid\langle V \cdot \mathbf{n} \phi\rangle_{K L}^{n}, \quad \forall L \in \partial K_{n}^{-} .
$$

Consequently, the corresponding terms in the definitions of $\mu_{h}$ and $\nu_{h}$ vanish and the equalities of the Lemma hold with

$$
\mathcal{M}_{N}^{1}:=\left\{K_{n} \in \mathcal{M}_{N}: K_{n} \cap \mathcal{B} \neq \emptyset\right\}
$$

We now estimate the $(d+1)$-dimensional Lebesgue measure of the union of the cells $K_{n} \in \mathcal{M}_{N}^{1}$. From the definition of $\phi$, for every $K_{n} \in \mathcal{M}_{N}^{1}$, there exist $(x, s) \in K_{n}$ such that $d(X(x, s), \partial A) \leq t^{1 / 2} h^{1 / 2}$. Since $\partial A=\cup_{1 \leq i \leq m} K_{i}^{\prime}$ । $L_{i}^{\prime}$, for every $K_{n} \in \mathcal{M}_{N}^{1}$, there exist $\left(x_{K, n}, s_{K_{n}}\right) \in K_{n}$ and $1 \leq i \leq m$ such that $d\left(X\left(x_{K_{n}}, s_{K_{n}}\right), x_{i}\right) \leq 2 t^{1 / 2} h^{1 / 2}$, where $x_{i}$ is the centroid of $K_{i}^{\prime}$ । $L_{i}^{\prime}$. Thus using the estimates of (1.6) on $\nabla Y$ and $\partial_{t} Y$, we obtain

$$
\begin{aligned}
\bigcup_{K_{n} \in \mathcal{M}_{N}^{1}}\left(x_{K_{n}}, s_{K_{n}}\right) & \subset \bigcup_{i=1}^{m} \bigcup_{0 \leq s \leq t} Y\left(B\left(x_{i}, 2 t^{1 / 2} h^{1 / 2}\right), s\right) \times\{s\} . \\
& \left.\subset \bigcup_{i=1}^{m} \bigcup_{n=0}^{N} B\left(Y\left(x_{i}, n \delta t\right), 2 C_{0} t^{1 / 2} h^{1 / 2}+C_{0} \delta t\right)\right) \times[n \delta t,(n+1) \delta t]
\end{aligned}
$$

and

$$
\left.\bigcup_{K_{n} \in \mathcal{M}_{N}^{1}} K_{n} \subset \bigcup_{i=1}^{m} \bigcup_{n=0}^{N} B\left(Y\left(x_{i}, n \delta t\right), 2 C_{0} t^{1 / 2} h^{1 / 2}+C_{0} \delta t+h\right)\right) \times[n \delta t,(n+1) \delta t] .
$$

Finally, using $C_{0} \geq 1, \delta t \leq c_{0} h$ and $h \leq c_{1} t$, we get

$$
\sum_{K_{n} \in \mathcal{M}_{N}^{1}} \delta t|K| \leq C C_{0}^{d} m t\left(t^{d / 2} h^{d / 2}+h^{d}\right) \leq C C_{0}^{d}\left\|u_{0}\right\|_{T V} t^{3 / 2} h^{1 / 2} .
$$

\section{$4 \quad$ Energy Estimates}

The two next lemmas contain the second key idea of the paper. The first one shows that the $L^{1}$-error at time $t$ controls $\mathcal{E}_{h}\left(u_{0}, t\right)$ (the jump of the $L^{2}$-energy of the approximate solution). The second one relates $\mathcal{E}_{h}\left(u_{0}, t\right)$ to the regularity of the approximate solution.

Lemma 4.1. We have

$$
\mathcal{E}_{h}\left(u_{0}, t\right) \leq 2\left\|\left(u_{h}-u\right)(\cdot, t)\right\|_{L^{1}} .
$$


Proof of Lemma 4.1: Using the conservation property of Corollary 1.2 with $f(v)=v^{2}$, we have $\|u(\cdot, t)\|_{L^{2}}^{2}=\left\|u_{0}\right\|_{L^{2}}^{2}$. Thus

$$
\mathcal{E}_{h}\left(u_{0}, t\right)=\left(\left\|u_{h}(\cdot, 0)\right\|_{L^{2}}^{2}-\left\|u_{0}\right\|_{L^{2}}^{2}\right)+\left(\|u(\cdot, t)\|_{L^{2}}^{2}-\left\|u_{h}(\cdot, t)\right\|_{L^{2}}^{2}\right) .
$$

Since $u_{h}(\cdot, 0)$ is the $L^{2}$-projection of $u_{0}$ on the mesh $\mathcal{T}$, the first term is non positive. Finally, $0 \leq u, u_{h} \leq 1$ yields $u^{2}(\cdot, t)-u_{h}^{2}(\cdot, t) \leq 2\left|u_{h}(\cdot, t)-u(\cdot, t)\right|$.

In particular, (3.12) implies

$$
\mathcal{E}_{h}\left(u_{0}, t\right) \leq 2 \mu_{h}(\phi)+2 \nu_{h}(\phi)+C\left\|u_{0}\right\|_{T V} t^{1 / 2} h^{1 / 2} .
$$

Lemma 4.2. We have the following identity

$$
\begin{aligned}
\mathcal{E}_{h}\left(u_{0}, t\right)=\sum_{K_{n} \in \mathcal{M}_{N}} \sum_{L \in \partial K_{n}^{-}}\left(1+\sum_{M \in \partial K_{n}^{-}}\right. & \left.\frac{V_{K M}^{n} \delta t}{|K|}\right)\left|V_{K L}^{n}\right| \delta t\left(u_{L}^{n}-u_{K}^{n}\right)^{2} \\
& +\frac{1}{2} \sum_{K_{n} \in \mathcal{M}_{N}} \sum_{L, M \in \partial K_{n}^{-}} \frac{V_{K L}^{n} V_{K M}^{n} \delta t^{2}}{|K|}\left(u_{M}^{n}-u_{L}^{n}\right)^{2} .
\end{aligned}
$$

Remark 4.1. We say that the identity of Lemma 4.2 is an Energy estimate, because of the analogy with the a priori inequality

$$
\left\|u_{\nu}(\cdot, 0)\right\|_{L^{2}}^{2}-\left\|u_{\nu}(\cdot, t)\right\|_{L^{2}}^{2} \leq 2 \nu \int_{0}^{t} \int_{\mathbf{R}^{d}}\left|\nabla u_{\nu}(x, s)\right|^{2} d x d s,
$$

where $u_{\nu}$ satisfies the advection-diffusion equation $\partial_{t} u_{\nu}+\operatorname{div}\left(V u_{\nu}\right)-\nu \Delta u_{\nu}=0$. In our case the diffusion is due to the scheme (and does not have the regularity and the isotropy of the Laplace operator), and the corresponding parameter $\nu$ is of order $h$.

Proof of Lemma 4.2: Let $n \geq 0$. Using Lemma 2.2 to change the index of summation in the last sum of the right hand-side of the first equality, we have

$$
\begin{aligned}
\sum_{K \in \mathcal{T}}|K|\left(u_{K}^{n}\right)^{2} & =\sum_{K \in \mathcal{T}}\left(|K|+\sum_{L \in \partial K_{n}^{-}} V_{K L}^{n} \delta t\right)\left(u_{K}^{n}\right)^{2}-\sum_{K \in \mathcal{T}} \sum_{L: K \in \partial L_{n}^{-}} V_{L K}^{n} \delta t\left(u_{K}^{n}\right)^{2} \\
& =\sum_{K \in \mathcal{T}}\left\{\left(|K|+\sum_{L \in \partial K_{n}^{-}} V_{K L}^{n} \delta t\right)\left(u_{K}^{n}\right)^{2}-\sum_{L \in \partial K_{n}^{-}} V_{K L}^{n} \delta t\left(u_{L}^{n}\right)^{2}\right\} \\
& =\sum_{K \in \mathcal{T}}|K| \sum_{L \in \mathcal{V}_{n}^{-}(K)} a_{K L}^{n}\left(u_{L}^{n}\right)^{2},
\end{aligned}
$$

where we have set for every $K_{n} \in \mathcal{M}, \mathcal{V}_{n}^{-}(K):=\partial K_{n}^{-} \cup\{K\}$, and for every $L \in \partial K_{n}^{-}$:

$$
a_{K K}^{n}:=1+\sum_{M \in \partial K_{n}^{-}} \frac{V_{K M}^{n} \delta t}{|K|}, \quad a_{K L}^{n}:=-\frac{V_{K L}^{n} \delta t}{|K|} .
$$


With this notations, (1.12) reads

$$
u_{K}^{n+1}=\sum_{L \in \mathcal{V}_{n}^{-}(K)} a_{K L}^{n} u_{K}^{n}
$$

for every $K_{n} \in \mathcal{M}$, and we have

$$
\begin{array}{r}
\sum_{K \in \mathcal{T}}|K|\left(\left(u_{K}^{n}\right)^{2}-\left(u_{K}^{n+1}\right)^{2}\right)=\sum_{K \in \mathcal{T}}|K|\left\{\sum_{L \in \mathcal{V}_{n}^{-}(K)} a_{K L}^{n}\left(u_{L}^{n}\right)^{2}-\left(\sum_{L \in \mathcal{V}_{n}^{-}(K)} a_{K L}^{n} u_{L}^{n}\right)^{2}\right\} \\
=\sum_{K \in \mathcal{T}}|K|\left\{\sum_{L \in \mathcal{V}_{n}^{-}(K)} a_{K L}^{n}\left(1-a_{K L}^{n}\right)\left(u_{L}^{n}\right)^{2}-\sum_{L, M \in \mathcal{V}_{n}^{-}(K), M \neq L} a_{K L}^{n} a_{K M}^{n} u_{L}^{n} u_{M}^{n}\right\} .
\end{array}
$$

Using the identity $\sum_{L \in \mathcal{V}_{n}^{-}(K)} a_{K L}^{n}=1$, we compute

$$
\begin{aligned}
\sum_{K \in \mathcal{T}}|K|\left(\left(u_{K}^{n}\right)^{2}-\left(u_{K}^{n+1}\right)^{2}\right) & =\sum_{K \in \mathcal{T}}|K|\left\{\sum_{L \in \mathcal{V}_{n}^{-}(K)} a_{K L}^{n} u_{L}^{n}\left(\sum_{M \in \mathcal{V}_{n}^{-}(K)} a_{K M}^{n}\left(u_{L}^{n}-u_{M}^{n}\right)\right)\right\} \\
& =\frac{1}{2} \sum_{K \in \mathcal{T}}|K| \sum_{L, M \in \mathcal{V}_{n}^{-}(K)} a_{K L}^{n} a_{K M}^{n}\left(u_{L}^{n}-u_{M}^{n}\right)^{2} .
\end{aligned}
$$

Summing on $0 \leq n \leq N$, we get the Lemma.

\section{Proof of the first part of Theorem 2}

In this section, we assume that $\xi>0$. We estimate successively $\mu_{h}(\phi)$ and $\nu_{h}(\phi)$. Applying the Cauchy-Schwarz inequality to the first formula of Lemma 3.5, we obtain

$$
\left|\mu_{h}(\phi)\right| \leq E_{h}\left(u_{0}, t\right)^{1 / 2}\left(\sum_{K_{n} \in \mathcal{M}_{N}^{1}}|K| \delta t^{2}\left(\frac{\langle\phi\rangle_{K}^{n}-\langle\phi\rangle_{K}((n+1) \delta t)}{\delta t}\right)^{2}\right)^{1 / 2}
$$

On the basis of (3.7) and the CFL condition (1.10) $\delta t \leq c_{0} h$, we have

$$
\left|\langle\phi\rangle_{K}^{n}-\langle\phi\rangle_{K}((n+1) \delta t)\right| \leq\left\|\partial_{t} \phi\right\|_{L^{\infty}} \delta t \leq C C_{0} \delta t t^{-1 / 2} h^{-1 / 2} \leq C C_{0} h^{1 / 2} t^{-1 / 2}
$$

and therefore, by (3.13)

$$
\left|\mu_{h}(\phi)\right| \leq C C_{0}^{1+d / 2} E_{h}\left(u_{0}, t\right)^{1 / 2}\left\|u_{0}\right\|_{T V}^{1 / 2} t^{1 / 4} h^{1 / 4} .
$$


We now write

$$
\begin{aligned}
\nu_{h}(\phi)= & \sum_{K_{n} \in \mathcal{M}_{N}^{1}} \sum_{L \in \partial K_{n}^{-}} \delta t\left(u_{L}^{n}-u_{K}^{n}\right) V_{K L}^{n}\left(\langle\phi\rangle_{K}^{n}-\langle\phi\rangle_{K L}^{n}\right) \\
& +\sum_{K_{n} \in \mathcal{M}_{N}^{1}} \sum_{L \in \partial K_{n}^{-}} \delta t\left(u_{L}^{n}-u_{K}^{n}\right)|K| L \mid\left(\langle V \cdot \mathbf{n}\rangle_{K L}^{n}\langle\phi\rangle_{K L}^{n}-\langle V \cdot \mathbf{n} \phi\rangle_{K L}^{n}\right), \\
= & : \text { I }+ \text { II. }
\end{aligned}
$$

From the Cauchy-Schwarz inequality, we have

$$
|\mathrm{I}| \leq E_{h}\left(u_{0}, t\right)^{1 / 2}\left(\sum_{K_{n} \in \mathcal{M}_{N}^{1}} \sum_{L \in \partial K_{n}^{-}}\left|V_{K L}^{n}\right| \delta t h^{2}\left(\frac{\langle\phi\rangle_{K}^{n}-\langle\phi\rangle_{K L}^{n}}{h}\right)^{2}\right)^{1 / 2}
$$

The conditions (1.8) implies

$$
\sum_{L \in \partial K_{n}^{-}}\left|V_{K L}^{n}\right| h \leq \alpha^{-2}\|V\|_{\infty}|K| \leq C|K|, \quad \forall K_{n} \in \mathcal{M}
$$

Consequently, (3.6) and (3.13) lead to

$$
|\mathrm{I}| \leq C C_{0}^{1+d / 2} E_{h}\left(u_{0}, t\right)^{1 / 2}\left\|u_{0}\right\|_{T V}^{1 / 2} t^{1 / 4} h^{1 / 4}
$$

We now estimate the term II; notice that it vanishes in case $V=$ Cst. Let $K_{n} \in \mathcal{M}_{N}^{1}$ and $L \in \partial K_{n}^{-}$, since $f_{K I L_{n}}\langle V \cdot \mathbf{n}\rangle_{K L}^{n}-V \cdot \mathbf{n}_{K L}=0$, we have

$$
\begin{aligned}
\langle V \cdot \mathbf{n}\rangle_{K L}^{n}\langle\phi\rangle_{K L}^{n}-\langle V \cdot \mathbf{n} \phi\rangle_{K L}^{n} & =f_{K I L_{n}} \phi(x, r)\left(\langle V \cdot \mathbf{n}\rangle_{K L}^{n}-V(x, r) \cdot \mathbf{n}_{K L}\right) d x d r \\
& =f_{K L_{n}}\left(\phi(x, r)-\langle\phi\rangle_{K L}^{n}\right)\left(\langle V \cdot \mathbf{n}\rangle_{K L}^{n}-V(x, r) \cdot \mathbf{n}_{K L}\right) d x d r .
\end{aligned}
$$

Using (3.6)-(3.7) and $\left|\langle V \cdot \mathbf{n}\rangle_{K L}^{n}-V(x, r) \cdot \mathbf{n}_{K L}\right| \leq C h$ for $(x, r) \in K_{।} L_{n}$, we get

$$
\left|\langle V \cdot \mathbf{n}\rangle_{K L}^{n}\langle\phi\rangle_{K L}^{n}-\langle V \cdot \mathbf{n} \phi\rangle_{K L}^{n}\right| \leq C C_{0} t^{-1 / 2} h^{3 / 2}
$$

Thus, we have

$$
|\mathrm{II}| \leq C C_{0}\left(\sum_{K_{n} \in \mathcal{M}_{N}^{1}}\left(\sum_{L \in \partial K_{n}^{-}}\left|K_{\mid} L\right| h\right) \delta t\right) t^{-1 / 2} h^{1 / 2}
$$

As above, for every $K_{n} \in \mathcal{M}$ we have $\sum_{L \in \partial K_{n}^{-}}\left|K_{।} L\right| h \leq C|K|$ and from (3.13):

$$
|\mathrm{II}| \leq C C_{0}^{d+1}\left\|u_{0}\right\|_{T V} t h
$$


Summing up (5.1)-(5.2)-(5.3), we obtain

$$
\left|\mu_{h}(\phi)\right|+\left|\nu_{h}(\phi)\right| \leq C\left(C_{0}^{d / 2+1} E_{h}\left(u_{0}, t\right)^{1 / 2}\left\|u_{0}\right\|_{T V}^{1 / 2} t^{1 / 4} h^{1 / 4}+C_{0}^{d+1}\left\|u_{0}\right\|_{T V} t h\right),
$$

and (4.1) yields

$$
\mathcal{E}_{h}\left(u_{0}, t\right) \leq C\left(C_{0}^{d / 2+1} E_{h}\left(u_{0}, t\right)^{1 / 2}\left\|u_{0}\right\|_{T V}^{1 / 2} t^{1 / 4} h^{1 / 4}+\left\|u_{0}\right\|_{T V}\left(t^{1 / 2} h^{1 / 2}+C_{0}^{d+1} t h\right)\right) .
$$

The following Lemma will allow us to bound $E_{h}\left(u_{0}, t\right)$ by $\mathcal{E}_{h}\left(u_{0}, t\right)$.

\section{Lemma 5.1.}

$$
E_{h}\left(u_{0}, t\right) \leq C \xi^{-1} \mathcal{E}_{h}\left(u_{0}, t\right) .
$$

Proof of Lemma 5.1: From (1.12) and the Cauchy-Schwarz inequality, we have for every $K_{n} \in \mathcal{M}$,

$$
|K|\left|u_{K}^{n+1}-u_{K}^{n}\right|^{2} \leq\left(\sum_{L \in \partial K_{n}^{-}} \frac{\left|V_{K L}^{n}\right| \delta t}{|K|}\right) \sum_{L \in \partial K_{n}^{-}}\left|V_{K L}^{n}\right| \delta t\left|u_{L}^{n}-u_{K}^{n}\right|^{2} .
$$

Due to the CFL condition (1.9), we have $\sum_{L \in \partial K_{n}^{-}}\left|V_{K L}^{n}\right| \delta t \leq|K|$; consequently, summing the preceding inequality on $K_{n} \in \mathcal{M}_{N}$, we obtain

$$
E_{h}\left(u_{0}, t\right) \leq 2 \sum_{K_{n} \in \mathcal{M}_{N}} \sum_{L \in \partial K_{n}^{-}}\left|V_{K L}^{n}\right| \delta t\left|u_{L}^{n}-u_{K}^{n}\right|^{2} .
$$

Using again the CFL condition, for every $K_{n} \in \mathcal{M}$ we have $\left(1+\sum_{M \in \partial K_{n}^{-}} V_{K M} \delta t /|K|\right) \geq \xi$. Thus $\xi E_{h}\left(u_{0}, t\right) / 2$ is bounded by the first sum of the right hand side of (4.2).

Plugging this inequality in (5.5), we get

$$
\mathcal{E}_{h}\left(u_{0}, t\right) \leq C\left(C_{0}^{d / 2+1} \mathcal{E}_{h}\left(u_{0}, t\right)^{1 / 2} \xi^{-1 / 2}\left\|u_{0}\right\|_{T V}^{1 / 2} t^{1 / 4} h^{1 / 4}+\left\|u_{0}\right\|_{T V}\left(t^{1 / 2} h^{1 / 2}+C_{0}^{d+1} t h\right)\right) .
$$

We then use the Young inequality $a b \leq \frac{1}{2} a^{2}+\frac{1}{2} b^{2}$ with

$$
a=C^{-1 / 2} \mathcal{E}_{h}\left(u_{0}, t\right)^{1 / 2}, \quad b=C^{1 / 2} C_{0}^{d / 2+1} \xi^{-1 / 2}\left\|u_{0}\right\|_{T V}^{1 / 2} t^{1 / 4} h^{1 / 4}
$$

to derive the estimate

$$
\mathcal{E}_{h}\left(u_{0}, t\right) \leq C C_{0}^{d+2}\left\|u_{0}\right\|_{T V}\left(\xi^{-1} t^{1 / 2} h^{1 / 2}+t h\right) .
$$

Finally, from Lemma 5.1 and the inequalities (5.4), (3.12), we compute successively

$$
\begin{aligned}
E_{h}\left(u_{0}, t\right) & \leq C C_{0}^{d+2} \xi^{-2}\left\|u_{0}\right\|_{T V}\left(t^{1 / 2} h^{1 / 2}+\xi t h\right), \\
\left|\mu_{h}(\phi)\right|+\left|\nu_{h}(\phi)\right| & \leq C C_{0}^{d+2} \xi^{-1}\left\|u_{0}\right\|_{T V}\left(t^{1 / 2} h^{1 / 2}+\xi^{1 / 2} t h\right), \\
\left\|\left(u_{h}-u\right)(\cdot, t)\right\|_{L^{1}} & \leq C C_{0}^{d+2} \xi^{-1}\left\|u_{0}\right\|_{T V}\left(t^{1 / 2} h^{1 / 2}+\xi^{1 / 2} t h\right) .
\end{aligned}
$$




\section{Proof of the Second part of Theorem 2}

We suppose that $V$ does not depend on the time variable. If

$$
\sup _{K \in \mathcal{T}}|K|^{-1} \sum_{L \in \partial K_{0}^{-}}\left|V_{K L}^{0}\right| \delta t<2 / 3
$$

then the proof of the first case allows us to conclude; therefore we can suppose that we have $\left|K^{\star}\right| \leq 2 \sum_{L \in \partial K_{0}^{\star,-}}\left|V_{K^{\star} L}^{0}\right| \delta t$ for some $K^{\star} \in \mathcal{T}$. This cell $K^{\star}$ satisfying (1.8), we deduce that the following inverse CFL condition holds:

$$
h \leq 2 \alpha^{-2}\|V\|_{\infty} \delta t .
$$

We now bound $\mu_{h}(\phi)$ and $\nu_{h}(\phi)$. From Lemma 3.5 and the estimate (3.7) on $\partial_{t} \phi$, we have

$$
\left|\mu_{h}(\phi)\right| \leq C \delta t\left(t^{-1 / 2} h^{-1 / 2}\right) \sum_{K_{n} \in \mathcal{M}_{N}^{1}}|K|\left|u_{K}^{n+1}-u_{K}^{n}\right| .
$$

Since $V$ does not depend on $t$, we have $V_{K}^{n}=V_{K}^{0}$, for every $K_{n} \in \mathcal{M}$. Consequently the discrete time derivative $v_{K}^{n}:=\delta t^{-1}\left(u_{K}^{n+1}-u_{K}^{n}\right)$ satisfies the scheme (1.12). The $L^{1}$-stability of the scheme and Lemma 2.1 imply

$$
\begin{aligned}
\sum_{K_{n} \in \mathcal{M}_{N}}|K|\left|u_{K}^{n+1}-u_{K}^{n}\right| & \leq \frac{t}{\delta t} \sum_{K \in \mathcal{T}}|K|\left|u_{K}^{1}-u_{K}^{0}\right| \leq t \sum_{K \in \mathcal{T}}\left|V_{K L}^{0}\right|\left|u_{K}^{0}-u_{L}^{0}\right| \\
& \leq t\|V\|_{\infty} \sum_{K \in \mathcal{T}}|K| L|| u_{K}^{0}-u_{L}^{0} \mid \leq C\left\|u_{0}\right\|_{T V} t
\end{aligned}
$$

Thus (using the CFL condition $\delta t \leq c_{0} h$ ), we have

$$
\left|\mu_{h}(\phi)\right| \leq C\left\|u_{0}\right\|_{T V} t^{1 / 2} h^{1 / 2}
$$

As in the proof of the first case (see (5.1)), we write $\nu_{h}(\phi)=\mathrm{I}+\mathrm{II}$. Recall that

$$
\mathrm{I}=\sum_{K_{n} \in \mathcal{M}_{N}^{1}} \sum_{L \in \partial K_{n}^{-}} \delta t\left(u_{L}^{n}-u_{K}^{n}\right) V_{K L}^{n}\left(\langle\phi\rangle_{K}^{n}-\langle\phi\rangle_{K L}^{n}\right)
$$

and that, from the estimate (3.6) on $\nabla \phi$, we have

$$
|\mathrm{I}| \leq C C_{0} h^{1 / 2} t^{-1 / 2} \sum_{K_{n} \in \mathcal{M}_{N}^{1}} \sum_{L \in \partial K_{n}^{-}} \delta t\left|V_{K L}^{n}\right|\left|u_{L}^{n}-u_{K}^{n}\right|
$$

Using the definition of the scheme (1.12), for $K_{n} \in \mathcal{M}_{N}^{1}$ and $L \in \partial K_{n}^{-}$, we have

$$
u_{L}^{n}-u_{K}^{n}=\frac{|K|}{\sum_{M \in \partial K_{n}^{-}} V_{K M}^{n} \delta t}\left(u_{K}^{n+1}-u_{K}^{n}\right)+\frac{1}{\sum_{M \in \partial K_{n}^{-}} V_{K M}^{n} \delta t} \sum_{M \in \partial K_{n}^{-}} V_{K M}^{n} \delta t\left(u_{L}^{n}-u_{M}^{n}\right) .
$$


Therefore,

$$
\begin{aligned}
&|\mathrm{I}| \leq C C_{0} h^{1 / 2} t^{-1 / 2}\left(\sum_{K_{n} \in \mathcal{M}_{N}^{1}}|K|\left|u_{K}^{n+1}-u_{K}^{n}\right|\right. \\
&\left.+\sum_{K_{n} \in \mathcal{M}_{N}^{1}} \frac{1}{\sum_{M \in \partial K_{n}^{-}}\left|V_{K M}^{n}\right| \delta t} \sum_{L, M \in \partial K_{n}^{-}} V_{K L}^{n} V_{K M}^{n} \delta t^{2}\left|u_{M}^{n}-u_{L}^{n}\right|\right) \\
&=C C_{0} h^{1 / 2} t^{-1 / 2}\left(\mathrm{I}_{1}+\mathrm{I}_{2}\right) .
\end{aligned}
$$

Using again the $L^{1}$-stability of the scheme, we have

$$
\left|\mathrm{I}_{1}\right| \leq C\left\|u_{0}\right\|_{T V} t
$$

For $\mathrm{I}_{2}$, we use the Cauchy-Schwarz inequality to get

$$
\begin{aligned}
\left|\mathrm{I}_{2}\right| \leq & \left(\sum_{K_{n} \in \mathcal{M}_{N}} \sum_{L, M \in \partial K_{n}^{-}} \frac{V_{K L}^{n} V_{K M}^{n} \delta t^{2}}{|K|}\left(u_{M}^{n}-u_{L}^{n}\right)^{2}\right)^{1 / 2} \\
& \times\left(\sum_{K_{n} \in \mathcal{M}_{N}^{1}} \sum_{L, M \in \partial K_{n}^{-}} \frac{V_{K L}^{n} V_{K M}^{n} \delta t^{2}|K|}{\left(\sum_{M \in \partial K_{n}^{-}} V_{K M}^{n} \delta t\right)^{2}}\right)^{1 / 2} \\
= & \left(\sum_{K_{n} \in \mathcal{M}_{N}} \sum_{L, M \in \partial K_{n}^{-}} \frac{V_{K L}^{n} V_{K M}^{n} \delta t^{2}}{|K|}\left(u_{M}^{n}-u_{L}^{n}\right)^{2}\right)^{1 / 2}\left(\sum_{K_{n} \in \mathcal{M}_{N}^{1}}|K|\right)^{1 / 2}
\end{aligned}
$$

Thanks to Lemma 4.2 , the first term is bounded by $\sqrt{2} \mathcal{E}_{h}\left(u_{0}, t\right)^{1 / 2}$ and by $(3.13)$, the second term is bounded by $C C_{0}^{d / 2}\left\|u_{0}\right\|_{T V}^{1 / 2} t^{3 / 4} h^{1 / 4} \delta t^{-1 / 2}$. Finally, using the inverse CFL condition (6.1), $\left|\mathrm{I}_{2}\right|$ is bounded by $C C_{0}^{d / 2}\left\|u_{0}\right\|_{T V}^{1 / 2} t^{1 / 4} h^{1 / 4} \mathcal{E}_{h}\left(u_{0}, t\right)^{1 / 2}$ and we have

$$
|\mathrm{I}| \leq C C_{0}^{d / 2+1}\left(\left\|u_{0}\right\|_{T V} t^{1 / 2} h^{1 / 2}+\mathcal{E}_{h}\left(u_{0}, t\right)^{1 / 2}\left\|u_{0}\right\|_{T V}^{1 / 2} t^{1 / 4} h^{1 / 4}\right) .
$$

The proof of the estimate (5.3) of the previous Section is still valid and we have $|\mathrm{II}| \leq$ $C C_{0}^{d+1}\left\|u_{0}\right\|_{T V}$ th. Summing up this estimate, (6.2) and (6.3), we obtain

$$
\begin{aligned}
& \left|\mu_{h}(\phi)\right|+\left|\nu_{h}(\phi)\right| \\
& \quad \leq C C_{0}^{d / 2+1}\left(\left\|u_{0}\right\|_{T V}\left(t^{1 / 2} h^{1 / 2}+C_{0}^{d / 2} t h\right)+\mathcal{E}_{h}\left(u_{0}, t\right)^{1 / 2}\left\|u_{0}\right\|_{T V}^{1 / 2} t^{1 / 4} h^{1 / 4}\right) .
\end{aligned}
$$

Plugging (6.4) in (4.1) and using Young inequality to absorb the term $\mathcal{E}_{h}\left(u_{0}, t\right)$ in the left hand side, we are led to

$$
\mathcal{E}_{h}\left(u_{0}, t\right) \leq C C_{0}^{d+2}\left\|u_{0}\right\|_{B V}\left(t^{1 / 2} h^{1 / 2}+t h\right) .
$$

The second part of the Theorem follows from the last estimate, (6.4) and (3.12). 


\section{References}

[BGP05] D. Bouche, J.-M. Ghidaglia, and F. Pascal, Error estimate and the geometric corrector for the upwind finite volume method applied to the linear advection equation, preprint (2005).

[Bre84] Y. Brenier, Averaged multivalued solutions for scalar conservation laws, SIAM J. Numer. Anal. 21 (1984), no. 6, 1013-1037.

[CCL94] B. Cockburn, F. Coquel, and P. LeFloch, An error estimate for finite volume methods for multidimensional conservation laws, Math. Comp. 63 (1994), no. $207,77-103$.

[CGY98] B. Cockburn, P.-A. Gremaud, and J. X. Yang, A priori error estimates for numerical methods for scalar conservation laws. III. Multidimensional fluxsplitting monotone schemes on non-Cartesian grids, SIAM J. Numer. Anal. 35 (1998), no. 5, 1775-1803 (electronic).

[CT80] M.G. Crandall and L. Tartar, Some relations between nonexpansive and order preserving mappings, Proc. Amer. Math. Soc. 78 (1980), no. 3, 385-390.

[Des04a] B. Després, Lax theorem and finite volume schemes, Math. Comp. 73 (2004), no. 247, 1203-1234 (electronic).

[Des04b] B. Després, An explicit a priori estimate for a finite volume approximation of linear advection on non-Cartesian grids, SIAM J. Numer. Anal. 42 (2004), no. 2, 484-504 (electronic).

[Des04c] B. Després, Convergence of non-linear finite volume schemes for linear transport, Notes from the XIth Jacques-Louis Lions Hispano-French School on Numerical Simulation in Physics and Engineering (Spanish). (2004), 219-239.

[EGH00] R. Eymard, T. Gallouët, and R. Herbin, Finite volume methods, Handbook of numerical analysis, Vol. VII, North-Holland, Amsterdam, 2000, pp. 713-1020.

[Fed69] H. Federer, Geometric measure theory, Die Grundlehren der mathematischen Wissenschaften, Band 153, Springer-Verlag New York Inc., New York, 1969.

[JP86] C. Johnson and J. Pitkäranta, An analysis of the discontinuous Galerkin method for a scalar hyperbolic equation, Math. Comp. 46 (1986), no. 173, 1-26.

[Kuz76] N. N. Kuznetsov, The accuracy of certain approximate methods for the computation of weak solutions of a first order quasilinear equation, Ž. Vyčisl. Mat. i Mat. Fiz. 16 (1976), no. 6, 1489-1502, 1627.

[OV04] M. Ohlberger and J. Vovelle, Error estimate for the approximation of non-linear conservation laws on bounded domains by the finite volume method, to appear in Math. of Comp. 
[Şab97] F. Şabac, The optimal convergence rate of monotone finite difference methods for hyperbolic conservation laws, SIAM J. Numer. Anal. 34 (1997), no. 6, 23062318.

[TS62] A. N. Tihonov and A. A. Samarskiü, Homogeneous difference schemes on irregular meshes, Ž. Vyčisl. Mat. i Mat. Fiz. 2 (1962), 812-832.

[TT95] T. Tang and Z. H. Teng, The sharpness of Kuznetsov's $O(\sqrt{\Delta x}) L^{1}$-error estimate for monotone difference schemes, Math. Comp. 64 (1995), no. 210, 581589.

[Vil94] J.-P. Vila, Convergence and error estimates in finite volume schemes for general multidimensional scalar conservation laws. I. Explicit monotone schemes, RAIRO Modél. Math. Anal. Numér. 28 (1994), no. 3, 267-295.

[VV03] J.-P. Vila and P. Villedieu, Convergence of an explicit finite volume scheme for first order symmetric systems, Numer. Math. 94 (2003), no. 3, 573-602. 